FORMATION Formation emploi

Revue française de sciences sociales

133 | Janvier-Mars 2016

Heurs et malheurs de l'apprentissage en Suisse

\title{
Aux origines de la fonction sociale de la formation professionnelle suisse
}

Une logique de reproduction sociale

The origins of the social aims of the Swiss VET system. A logic of social

reproductionDie Ursprung der sozialen Zielsetzungen des Schweizerischen

Berufsbildungssystems. Eine Logik der sozialen Reproduktion

En los orígenes de la función social de la formación profesional suiza : una lógica

de reproducción social

\section{Lorenzo Bonoli}

\section{(2) OpenEdition}

Journals

Édition électronique

URL : http://journals.openedition.org/formationemploi/4637

DOI : $10.4000 /$ formationemploi.4637

ISSN : 2107-0946

Éditeur

La Documentation française

Édition imprimée

Date de publication : 20 avril 2016

Pagination : 17-34

ISSN : 0759-6340

Référence électronique

Lorenzo Bonoli, «Aux origines de la fonction sociale de la formation professionnelle suisse »,

Formation emploi [En ligne], 133 | Janvier-Mars 2016, mis en ligne le 21 avril 2018, consulté le 30 octobre 2020. URL : http://journals.openedition.org/formationemploi/4637 ; DOI : https://doi.org/

10.4000/formationemploi.4637 


\title{
Aux origines de la fonction sociale de la formation professionnelle suisse \\ Une logique de reproduction sociale
}

\author{
LORENZO BONOLI \\ Maître de recherche et d'enseignement à l'Institut fédéral des hautes études \\ en formation professionnelle, Lausanne
}

Résumé

Aux origines de la fonction sociale de la formation professionnelle suisse. Une logique de reproduction sociale

La formation professionnelle suisse se développe, dans sa forme institutionnelle actuelle, à partir de la deuxième moitié du XIX ${ }^{\mathrm{e}}$ siècle, poursuivant deux finalités principales : l'une économique, assurer une main-d'œuvre qualifiée pour les entreprises, et l'autre sociale, œuvrer pour une stabilisation socio-économique de la société. Après avoir décrit les étapes de cette institutionnalisation, l'article analyse la finalité sociale qui lui avait été attribuée, en relevant la volonté d'amélioration des conditions de vie des classes populaires mais aussi une logique de reproduction socio-professionnelle. Cette dernière, progressivement, va laisser place à une volonté de promotion de la mobilité socio-professionnelle.

Mots clés : enseignement technique-professionnel, politique de l'éducation, inégalité sociale, reproduction sociale, Suisse

Abstract

The origins of the social aims of the Swiss VET system. A logic of social reproduction

The Swiss Vocational Education and Training (VET) system developed progressively during the second half of the XIX century, following two main aims: An economic aim, securing a qualified workforce for Swiss companies, and a social aim: the social-political stabilization of society. After a description of the most important steps of institutionalization, the article will analyze its social aims, highlighting an improvement in the living conditions of the lowest classes but also a logic of social reproduction. The social aims develop from a logic of social reproduction to today's logic of social mobility.

Keywords: technical \& vocational education, education policy, social inequality, social reproduction, Switzerland

Journal of Economic Literature: I 21, I 28, J 62

Traduction : Auteur 
La formation professionnelle ${ }^{1}$ suisse connaît, depuis quelques années, un grand succès. Elle est saluée par les experts et les politiciens, par les représentants du monde de l'économie et par les jeunes qui sont très nombreux à opter pour cette voie.

Deux caractéristiques du système suisse sont régulièrement mises en avant : tout d'abord, sa capacité à former des professionnels en lien avec les besoins des entreprises; ensuite, son aptitude à faciliter l'insertion des jeunes dans le monde du travail, en limitant ainsi les risques de chômage et d'inégalités sociales. Pourtant, à y regarder de près, force est de constater qu'une tension traverse ce système, entre finalités d'ordre économique et finalités d'ordre social.

Dès lors, il convient de s'interroger sur l'origine de cette tension qui confronte deux logiques souvent contradictoires ${ }^{2}$. Adoptant un point de vue historique, nous montrerons que cette tension existe dès les débuts de l'institutionnalisation du système de formation professionnelle, autour de 1900. Cependant, si les finalités économiques visant à répondre au besoin de main-d'œuvre qualifiée des entreprises ne sont pas fondamentalement différentes de celles évoquées aujourd'hui, d'importantes transformations ont en revanche marqué l'évolution des finalités sociales. Si aujourd'hui, l'objectif est une meilleure intégration et une réduction des inégalités sociales, autour de 1900, il était tout autre : assurer une stabilité socio-politique de la société via l'amélioration des conditions de vie des classes populaires, mais aussi via la reproduction des différences sociales de l'époque.

L'enjeu de cet article sera alors, tout d'abord, de fournir une brève reconstruction historique du processus d'institutionnalisation de la formation professionnelle suisse, qui a lieu entre 1880 et 1930 . Cette reconstruction soulignera surtout les finalités économiques motivant un tel processus. Ensuite, seront décrites les caractéristiques de la fonction sociale qui, dès son origine, est également attribuée à cette filière. Il s'agira notamment de relever que la formation professionnelle est explicitement considérée comme un outil incontournable pour réduire les tensions provoquées par ce qu'on appelait, à l'époque, la " question sociale ». Il apparaitra ainsi que cette filière est investie d'une double mission, caractérisée par une tension entre éléments progressistes et conservateurs : d'une part, améliorer les conditions de vie des classes populaires ; d'autre part, participer à la stabilisation

1. Par l'expression "formation professionnelle "(Berufsbildung, formazione professionale), la Suisse désigne la filière du système éducatif qui fait suite à l'école obligatoire et qui offre des formations initiales ou supérieures préparant directement à l'exercice d'un métier. Ces formations se déroulent dans des écoles professionnelles à plein temps et, surtout, dans le cadre d'un apprentissage en alternance ; celui-ci, s'agissant de la formation professionnelle initiale, est choisi par $64 \%$ des jeunes poursuivant une formation après l'école obligatoire. (SEFRI, 2015).

2. L'articulation entre finalités économiques et sociales recouvre en partie la tension " produire/former ", centrale dans les études sociologiques sur l'apprentissage, aussi bien dans le contexte français que suisse, ( $c f$. en particulier Moreau, 2003 et Lamamra, 2013). Cependant, ces deux finalités se situent à un niveau plus général, englobant non seulement la situation de l'apprenti, mais les finalités du système de formation professionnelle dans son ensemble. 
de la société et à la reproduction des inégalités sociales afin d'éviter tout bouleversement socio-politique majeur. Une telle exploration permettra de mettre en lumière le changement entre les finalités sociales de l'époque et celles d'aujourd'hui. Enfin, dans la conclusion, nous soulèverons la question de savoir jusqu'à quel point le système actuel a pu effectivement assimiler les nouvelles finalités d'intégration et de réduction des inégalités qui lui ont été assignées ces dernières décennies.

La reconstruction historique proposée ici s'appuie sur une analyse des discours portant sur des sources qui couvrent la période décisive dans le processus d'institutionnalisation de la formation professionnelle, soit de 1880 à 1930.

\section{Encadré 1 : Méthodologie et sources}

En tant qu'approche théorique et méthodologique, l'analyse des discours $\left({ }^{*}\right)$ met l'accent sur les discours produits, dans notre cas, par les acteurs de la formation professionnelle de l'époque, pour en relever les conditions de leur énonciation et de leur réception. Plus précisément, une telle analyse s'applique, premièrement, à relever ce que les acteurs de l'époque disaient à propos de la formation professionnelle et, deuxièmement, à expliquer pourquoi ils pouvaient dire cela : autrement dit, et dans le concret du thème ici approfondi, expliquer pourquoi, à un certain moment historique, en fonction de conditions socio-économiques déterminées, une série d'affirmations concernant le rôle de la formation professionnelle devient non seulement possible mais également courante, faisant ainsi apparaître une " conception » discursivement partagée de ce type de formation, et en particulier des attentes au niveau économique et social qui lui sont attribuées $\left(^{* *}\right)$.

(*) Pour une introduction à l'analyse des discours, cf. Maingueneau (1991). Les travaux de Michel Foucault (notamment 1966/1969) constituent des modèles importants pour la constitution d'une telle approche, qui connaît des développements récents dans le domaine des sciences sociales, notamment en Allemagne, cf. Keller (2011), Jäger (2012) et Füssel \& Neu (2014).

$\left.{ }^{* *}\right)$ Concrètement, l'analyse a été effectuée sur la base d'un corpus de textes comprenant des textes officiels (lois, messages gouvernementaux, rapports officiels), des publications (livres, revues, brochures) et articles de presse, publiés entre 1880 et 1930 . Un tel corpus donne accès aux positions de différents acteurs ayant pris part aux débats de l'époque (politiciens, journalistes, chercheurs universitaires, enseignants, fonctionnaires en charge de la formation professionnelle, représentants des milieux patronaux et des milieux ouvriers, etc.).

\section{Le développement du système suisse de formation professionnelle}

En Suisse, comme dans la plupart des pays européens ${ }^{3}$, le système de formation professionnelle se met en place sous sa forme institutionnelle actuelle vers la fin du XIX ${ }^{\mathrm{e}}$ siècle et début du XX $\mathrm{XX}^{\mathrm{e}}$. L'introduction de la liberté de commerce et d'entreprise ainsi que le

3. Cf. CEDEFOP (2004), pour la France nous renvoyons à Pelpel et Troger (2001). 
développement de l'industrialisation plongent les structures traditionnelles corporatives d'initiation des jeunes aux métiers dans une profonde crise tout au long du XIX ${ }^{e}$ siècle. Les réactions face à cette situation s'intensifient vers la fin du siècle, lorsque différents acteurs (notamment les associations professionnelles, le gouvernement fédéral et les gouvernements cantonaux) initient une série d'initiatives qui conduisent progressivement à l'adoption des premières réglementations dans le domaine de l'apprentissage et à la création des premières écoles professionnelles ( $c f$. Gonon, 1998 ; Bonoli, 2012).

Ces initiatives se développent dans un contexte institutionnel marqué par un fort fédéralisme et un appareil juridique fédéral en pleine élaboration. La Constitution fédérale en vigueur, adoptée en 1874, attribue aux 25 cantons qui, à l'époque, composent la Suisse, une grande autonomie législative, en particulier dans le domaine de l'instruction et dans la réglementation du secteur du commerce et des arts et métiers. Elle laisse de facto une marge de manœuvre limitée au gouvernement fédéral pour intervenir dans la formation professionnelle. Parallèlement, l'appareil juridique du jeune Etat suisse, qui nait dans son organisation moderne avec la Constitution de 1848, comporte de nombreux vides, notamment en matière de règlementation du travail dans le domaine des arts et métiers et dans les conditions de l'apprentissage d'un métier.

C'est dans ce contexte peu réglementé que les associations professionnelles du domaine des arts et métiers et du commerce prennent en charge la relance de la formation des jeunes. Ce sont sourtout des associations liées à des métiers ou secteurs d'activité professionnelle qui naissent vers la fin du XIX ${ }^{\mathrm{e}}$ siècle pour défendre leurs intérêts.

Concrètement, ces associations vont être à l'origine d'une série d'initiatives, d'abord à l'échelle locale, puis nationale, visant à relancer la formation professionnelle. Elles vont organiser, notamment, des cours professionnels du soir et du dimanche, établir des règlements d'apprentissage, introduire des examens de fin d'apprentissages dans certaines professions et faire pression sur les milieux politiques pour obtenir un soutien de l'Etat en faveur de ce type de formation et surtout pour inscrire sa réglementation dans une loi fédérale.

Le développement et l'institutionnalisation de la formation professionnelle ont toujours bénéficié d'un large soutien des associations professionnelles du secteur du commerce et des arts et métiers. Il s'agissait de secteurs composés avant tout de petites et moyennes entreprises qui, compte tenu de leurs dimensions, avaient tout intérêt à voir se développer un système public de formation professionnelle. Caractérisées par une automatisation réduite, ces entreprises considéraient les qualifications des travailleurs comme la seule réponse possible à la concurrence de l'étranger et de la grande industrie. Le soutien au développement d'un système public de formation professionnelle était en revanche plus nuancé dans les milieux de la grande industrie, souvent autonomes en ce qui concerne la formation de leur propre main-d'œuvre. Ils étaient en général plutôt hostiles aux nouvelles réglementations dans le domaine ( $c f$. Bauder, 2008). 
Ainsi, sous la pression d'associations professionnelles telle que l'Union suisse des arts et métiers (Usam), la formation professionnelle devient progressivement un thème de politique économique, mettant en évidence la relation entre une meilleure formation des artisans et une amélioration de la situation économique du pays, comme cela émerge clairement d'une publication de 1881 de l'Usam où l'on peut lire :

"Une meilleure formation des artisans et des patrons d'entreprise est d'une importance extrême pour l'efficience économique et par là pour le bien-être de notre peuple; elle est donc une exigence urgente et absolue. " (USAM-SGV 1881, p. 6)

Le fait de souligner une telle relation reflète la volonté d'une des associations professionnelles les plus influentes de l'époque d'offrir une justification aux instances politiques pour une intervention dans le domaine de la formation professionnelle. Et c'est sur la base d'une telle relation que le gouvernement fédéral pourra désigner explicitement la formation professionnelle comme un outil de politique économique ${ }^{4}$ et subventionner, à partir de 1884 , les institutions actives dans ce type d'enseignement.

Cependant, les compétences limitées de la Confédération ne permettent pas l'adoption de dispositions règlementant et organisant la filière au niveau national. Ainsi, les premières lois dans le domaine vont apparaître au niveau des cantons qui, à partir de 1890 , et de manière progressive, vont se doter de législations propres sur les apprentissages et sur la filière professionnelle en général.

Ce mouvement progressif d'institutionnalisation va aboutir, en 1930, à l'adoption de la première loi fédérale sur la formation professionnelle ; adoption rendue possible grâce à la modification de la Constitution de 1908 attribuant à la Confédération le droit de légiférer dans le domaine des arts et métiers et du commerce. Tout en s'inspirant des lois cantonales déjà existantes et avec le soutien décisif des milieux des arts et métiers et du commerce, cette première loi fédérale va uniformiser les dispositions concernant l'apprentissage et coordonner les différentes initiatives dans le domaine, offrant à la Suisse les bases pour l'établissement d'un système national de formation professionnelle, avec un diplôme de fin d'apprentissage reconnu et protégé sur tout le territoire. Nous sommes là à l'origine du Certificat fédéral de capacité (CFC), diplôme toujours existant et qui couronne aujourd'hui une formation professionnelle initiale de trois ou quatre ans ${ }^{5}$.

4. Cf. Conseil fédéral, 1883 , p. $657 / 658$.

5. Cf. Muriel Surdez (2005) pour une reconstruction de la mise en place de ce diplôme. 


\section{L'émergence de la «question sociale»}

La brève reconstruction du processus d'institutionnalisation proposée ici permet de révéler les motivations économiques qui accompagnent le développement de la filière, mais des débats de l'époque émerge une autre préoccupation : la formation professionnelle apparaît en effet également comme un outil pouvant participer à la résolution d'une série de problèmes sociaux apparus tout au long du XIX ${ }^{\mathrm{e}}$ siècle.

Sous l'effet des mutations engendrées par la Révolution française et la révolution industrielle, le XIX ${ }^{\mathrm{e}}$ siècle est une période de grands bouleversements et de grande instabilité socio-politique dans le monde du travail comme dans l'organisation de la vie quotidienne : d'une part, on assiste à la diffusion du travail salarié, à la prolétarisation progressive d'une partie de la population et à l'émergence de mouvements de protection des ouvriers et de contestation du système productif (avec une multiplication de manifestations, de grèves et de lock-out $t^{\dagger}$; d'autre part, on observe également un déplacement des populations paysannes vers les centres industriels, des changements dans les rapports familiaux avec des parents contraints à travailler loin de la maison et des enfants obligés d'aller à l'école et enfin une nouvelle structuration de la vie quotidienne, avec des horaires scandés par le travail salarié et l'apparition du " temps libre » et de ses conséquences : l'augmentation des auberges, les fêtes du dimanche, les problèmes d'alcoolisme, mais aussi un peu plus tard l'apparition des associations de loisirs, du cinéma et du sport.

Ces changements sont à l'origine d'une série de tensions au sein de la société de l'époque, qui seront désignées par l'expression " question sociale " ${ }^{7}$ : une expression qui recouvre ainsi aussi bien les problèmes liés aux conditions de vie et de travail d'une partie de la population que l'instabilité socio-politique engendrée par les mouvements de contestation et de défense des travailleurs.

6. Comme la plupart des pays européens, la Suisse connaît aussi, entre la fin du XIX et le début du XX ${ }^{e}$ siècle, une série importante de mouvements de contestation. Dans son ouvrage sur les conflits liés au monde du travail en Suisse, entre 1880 et 1914, Hans Hirter (1983) a répertorié plus de 2426 mouvements de grève repartis sur l'ensemble de la période étudiée. Les années 1906 et 1907, avec respectivement 265 et 282 grèves, sont de loin les plus tendues dans l'histoire du monde du travail suisse.

7. L'expression "question sociale ", ou "soziale Frage " en allemand, apparaît dans la deuxième moitié du $\mathrm{XIX}^{\mathrm{e}}$ siècle et s'impose, en Suisse, comme un concept incontournable pour toute réflexion sur la situation sociale et économique de l'époque ; et ce, aussi bien dans les discours des milieux bourgeois que dans ceux des représentants du mouvement ouvrier. Pour une contextualisation historique des enjeux principaux de la question sociale en Suisse, $c f$. Gruner, 1968, p. 15 ss et Widmer, 1995, p. 672 ss. 


\section{Une réponse à la « question sociale » entre conservatisme et progressisme}

S'adressant aux milieux ouvriers et du petit artisanat particulièrement touchés par ces changements, la formation professionnelle est traversée en profondeur par la question sociale. Elle sera ainsi régulièrement convoquée dans les débats où elle apparaîtra comme un outil de politique sociale en mesure d'atténuer la gravité des tensions et d'œuvrer ainsi à une stabilisation de la société.

Comme cela apparaîtra dans le choix de citations proposées par la suite, ce sont en particulier les milieux bourgeois ${ }^{8}$ qui font de ce type de formation un outil de résolution de la question sociale. De leur côté, les milieux de gauche et les syndicats, bien que profondément engagés dans les débats autour de la question sociale, ne se positionnent que rarement sur le thème de la formation professionnelle, laissant l'apanage des débats aux premiers. Deux précisions s'imposent à ce propos : d'une part, pendant la période étudiée, les préoccupations majeures des milieux de gauche et syndicaux sont liées à l'amélioration des conditions de travail (salaires, horaires, sécurité, hygiène, etc.), sans s'emparer clairement du thème de la formation des ouvriers; d'autre part, favorables en principe à une amélioration des conditions d'apprentissage d'une profession, ces milieux considèrent souvent la formation professionnelle comme une formation " patronale ", destinée à assurer la productivité des travailleurs. De ce fait, elle ne permet pas un «développement harmonieux de l'Homme ", mais tout au plus un "dressage » [Abrichterei] destiné à créer des " automates", (Arbeiterstimme, 19.2.1881).

Or, si dans les discours des milieux bourgeois, la filière professionnelle apparait, au tournant du $\mathrm{XX}^{\mathrm{e}}$ siècle, comme un outil incontournable pour répondre aux défis de la question sociale et œuvrer pour une stabilisation de la société, cette filière se voit chargée d'une double mission, sous certains aspects contradictoire.

Elle se retrouve en effet confrontée à un délicat exercice d'équilibriste entre, d'une part, la mission de confirmation et de reproduction d'une série de distinctions sociales qui ont structuré la société suisse jusqu'alors, et qui apparaissent comme les conditions mêmes de sa stabilisation et de son développement harmonieux et, de l'autre, la mission d'intégration, modérée et contrôlée, d'éléments nouveaux reflétant l'évolution de la société, et notamment les nouvelles aspirations d'égalité et de liberté héritées de la

8. Notamment les élites politiques d'orientation libérale et conservatrice, les fonctionnaires publics actifs dans le domaine de l'instruction, les représentants d'associations professionnelles patronales ou encore les journalistes écrivant pour des quotidiens d'orientation libérale ou conservatrice.

9. Sur le rôle « en retrait » des partis de gauche et des syndicats sur le thème de la formation professionnelle, des études plus approfondies restent encore à réaliser, $c f$. néanmoins T. Bauder (2008, p. 146) et J.-P. Tabin (1989, p. 94). 
Révolution française, ainsi que les espoirs de conditions de vie meilleures suscités par les progrès au niveau productif induits par la révolution industrielle ${ }^{10}$.

Comme nous le verrons, un tel exercice d'équilibriste transparaît des discours autour de la formation professionnelle. Il prend la forme d'une tension entre, d'une part, un certain progressisme, qui se traduit dans la volonté d'assurer une meilleure éducation des classes populaires et d'améliorer ainsi leurs conditions de vie ; et, d'autre part, un certain conservatisme, qui se concrétise dans la défense du système social et productif de l'époque et, en particulier, dans la volonté de reproduction des distinctions sociales qui le caractérisent. Plus précisément, cette tension apparaît dans les discours de l'époque entre la volonté de promouvoir l'égalité entre tous les citoyens et la justification de certaines différences "naturelles ", entre la volonté de promouvoir l'égalité de tous les citoyens face à l'instruction et la séparation nette entre filières destinées aux couches inférieures de la population et filières pour les élites et, enfin, entre la volonté d'améliorer les conditions de vie des classes populaires via une meilleure instruction et celle de ne pas bouleverser la structure sociale de l'époque.

\subsection{Un délicat équilibre entre promotion de l'égalité et confirmation des « différences naturelles »}

Léquilibre délicat entre éléments progressistes et éléments conservateurs représente une clé de lecture pertinente pour analyser les débats de l'époque.

L'ouvrage d'instruction civique pour l'enseignement secondaire et les écoles professionnelles complémentaires, publié en 1884 par Numa Droz ${ }^{11}$, est significatif à cet égard. Rédigé par un ministre du gouvernement fédéral en exercice, il présente les grands principes de la vie en société du point de vue officiel des élites politiques.

Droz souligne les améliorations en termes d'égalité et de liberté apportées par la Révolution française, mais en même temps, il confirme la plupart des distinctions structurant le tissu social de l'époque. Une tension entre éléments progressistes et conservateurs apparaît ainsi lors de la présentation de la notion d' " égalité ".

Si la notion est présentée comme un des piliers des jeunes démocraties, sa portée est fortement nuancée par le rappel des "différences naturelles entre les hommes et les peuples " qui impliquent qu'il y aura toujours « des êtres faibles pour lesquels les luttes de l'existence

10. En commentant les discours sur la crise économique qui touche la Suisse pendant les années 1880, Thomas Widmer (1992) montre de façon brillante le jeu d'équilibriste entre la volonté de maintenir l'ancien, seul élément de certitude dans une société en pleine évolution, et celle de profiter du " progrès » engendré par les nouvelles conditions politiques et productives.

11. Numa Droz, ministre du gouvernement fédéral de 1875 à 1898 , est un représentant du parti radical suisse, un parti d'orientation libérale, défendant des positions progressistes et favorables à une intervention modérée de l'Etat dans le domaine du travail et de l'instruction ( $c f$. Dictionnaire historique suisse, DHS). 
sont plus pénibles que pour d'autres " (p. 45). Droz rejette ainsi les thèses "chimériques" de ceux " connus [...] sous le nom de communistes et de nihilistes " qui aimeraient imposer par la violence l'égalité absolue entre les êtres humains pour défendre l' "égalité véritable " qui est l'égalité des droits, et notamment l'égalité devant la loi, l'instruction et le travail (pp. 47/48). Cependant, dans le discours de Droz, il s'agit d'une égalité formelle qui tolère, en réalité, des situations de vie très inégales émanant d'un " état naturel des choses » sur lequel l'homme et la société n'ont pas de prise.

\subsection{La formation professionnelle pour contribuer à l'égalité face à l'instruction}

Larticulation entre éléments progressistes et conservateurs émerge également des discours confrontant la formation professionnelle à la voie des études générales. Dans une période où les structures de la filière professionnelle sont encore en développement, de nombreux acteurs dénoncent "l'injustice sociale » qui veut que la formation pour les classes supérieures de la population soit très bien organisée alors que rien n'est fait pour les classes populaires.

Dans un esprit égalitaire, on réclame ainsi la mise en place d'écoles s'adressant également aux couches inférieures de la population ; cela est illustré par la prise de position de Léon Genoud, directeur de l'école d'arts et métiers (Technicum) de Fribourg ${ }^{12}$ :

"Il est douloureux de constater que [...] l'enfant de parents riches est protégé par la collectivité. Pour lui, tout est prévu : collèges, écoles secondaires, universités etc. ; que l'Etat entretient à grands frais. Pour l'enfant pauvre, rien de tout cela. Or, les enfants pauvres ou de condition modeste sont la majorité. Ils sont appelés à exercer les professions les plus nécessaires au point de vue économique ; il est donc urgent de prendre des mesures pour qu'ils apprennent ces métiers. " (Genoud, in ASCAPA, 1920, p. 25)

Cependant, dans cette citation, la dénonciation de l'inégalité de traitement entre « riches » et " pauvres » porte uniquement sur les infrastructures et non sur les curricula: en effet, la différence au niveau du type de formation entre les deux groupes n'est pas remise en question. Cette citation révèle ainsi comment une distinction censitaire entre " riches " et " pauvres " se traduit directement dans une distinction entre deux filières de formation séparées par une "barrière infranchissable " ${ }^{13}$ : pour les premiers, la formation générale, pour les deuxièmes, la formation professionnelle. Ainsi l'Etat, en promouvant la formation professionnelle, est appelé à contribuer à l'établissement d'une égalité face

12. Député conservateur au parlement cantonal fribourgeois, Léon Génoud sera un grand promoteur de la formation professionnelle. Fondateur du journal L'Artisan et de la Revue suisse d'enseignement professionnel, il dirige le Technicum de Fribourg entre 1896 et 1925, (Cf. DHS).

13. L'expression est de F. Guillermet, in Gazette de Lausanne, 06.07.1907. 
à l'instruction entre les classes; une égalité qui ne doit cependant pas aller jusqu'à remettre en question une telle barrière curriculaire.

\subsection{Une formation professionnelle qui assure la continuité socio-professionnelle}

Dans les débats de l'époque, la promotion de la filière répond non seulement à une volonté d'égalité face à l'instruction, mais aussi à une volonté d'améliorer les conditions de vie des classes populaires : amélioration centrée sur une meilleure insertion dans le monde du travail des jeunes issus de ces milieux, rendue possible par leur meilleure qualification.

C'est dans cette optique que l'on peut lire dans le Journal de Genève, des articles faisant de la formation professionnelle une "mesure préventive " contre les " misères sociales " (JdG, 02.08.1890) ou encore un moyen privilégié pour lutter contre le "paupérisme " ( $d d G$, 28.05.1891), assurant au jeune issu des classes populaires les instruments nécessaires pour "gagner sa vie honorablement» $(J d G, 02.08 .1882)$.

Mais en même temps, une telle amélioration des conditions de vie ne doit pas être à l'origine de changements majeurs au niveau de l'appartenance sociale des jeunes qui la suivent. Ainsi, dans les discours analysés, l'amélioration des conditions de vie des classes populaires est systématiquement nuancée par la volonté de défendre l'ordre social établi en respectant scrupuleusement une continuité entre origine sociale (classes populaires), type de formation (formation professionnelle), activité future (ouvrier ou artisan). Une telle continuité socioprofessionnelle constitue un présupposé essentiel dans les débats de l'époque. Elle s'impose comme une évidence, jamais remise en doute et surtout, comme toute évidence, ne faisant que rarement l'objet de motivations ou de justifications explicites.

En tant que présupposé discursif, cette continuité entre origine sociale et avenir professionnel sous-tend clairement les réflexions concernant l'organisation des écoles pour les classes populaires, et notamment l'établissement des programmes d'enseignement.

L'idée d'un enseignement uniforme pour tous les jeunes, même pendant l'école obligatoire, est fortement nuancée dans les réflexions de l'époque. S'agissant de l'école pour les classes populaires, ces réflexions vont dans le sens d'une meilleure adéquation entre les enseignements scolaires et les besoins futurs des jeunes, facilement établis en fonction de leur origine sociale :

"L'enseignement devrait préparer l'enfant à sa 'profession pour la vie' [Lebensberuf] ; pour cette raison, il ne peut pas être le même pour tous les enfants. Paysans, artisans et manouvres seraient autrement détournés [entfremdet] de leur profession. Le programme d'enseignement pour les enfants des classes des travailleurs devrait se limiter à ce que l'enfant apprenne à lire, écrire et calculer [...]. " (Basler Grenzpost, 1884, cit. In Widmer 1992, p. 235)

Cette logique de reproduction ne se limite pas à promouvoir la reproduction de la classe générique des "travailleurs ", mais va jusqu’à la volonté d'instaurer une continuité au 
niveau de l'activité professionnelle elle-même. A cet époque, en Suisse, la filière professionnelle s'adresse avant tout au milieu des arts et métiers, en particulier aux ouvriers de petites et moyennes entreprises, mais aussi souvent à des artisans exerçant leur propre activité indépendante.

Dans ce contexte, la volonté de reproduction socio-professionnelle va se traduire par l'importance accordée au fait que les jeunes issus de familles actives dans l'agriculture, dans le commerce, dans telle ou telle activité artisanale restent dans le même domaine. Cette logique émerge notamment des ouvrages d'orientation professionnelle où la possibilité de se former dans le même domaine d'activité des parents est fortement encouragée ${ }^{14}$.

Cette logique de reproduction socio-professionnelle répond directement à une préoccupation formulée dans le passage du Basler Grenzpost, cité précédemment : "Paysans, artisans et manouvres seraient autrement détournés de leur profession. " Autrement dit, au centre des craintes, se trouve un mouvement d'individus qui quittent les professions manuelles et agricoles pour s'orienter vers des activités de bureau ou même des professions libérales. Un mouvement encouragé apparemment par une école obligatoire jugée trop centrée sur des enseignements théoriques, éloignés de la réalité du travail. Léon Genoud (1901) déclarera à ce propos :

"Il ne faut pas oublier que si le jeune garçon doit être un ouvrier des champs ou de l'atelier, la première et la meilleure sauvegarde de sa moralité sera le goût et l'amour du travail qui le fera vivre plus tard."

"Or, l'Ecole actuelle élève les enfants comme sils devaient être de petits rentiers; elle les éloigne des professions manuelles pour les jeter dans les professions dites libérales au lieu de les élever pour qu'ils deviennent des hommes de progrès, des ouvriers habiles, assidus et ayant du gồt. " (1901, p. 408)

Les préoccupations liées à cette mobilité ascendante sont de deux ordres : l'un économique, l'autre socio-politique.

Tout d'abord, comme le relève Robert Comtesse (1890), Conseiller d'Etat du canton de Neuchâtel et futur président de la Confédération Helvétique ${ }^{15}$ :

"Notre intérêt économique bien compris n'est pas de pousser davantage nos jeunes gens dans le courant des professions libérales déjà très encombrées et d'augmenter la légion de ceux que les ambitions et les sacrifices de leurs parents, souvent bien mal inspirés, arrachent à l'agriculture, à l'industrie, aux métiers lucratifs de nos villes et de nos villages [...] Notre intérêt économique nous commande de nous préoccuper avant tout du recrutement de cette armée de travailleurs. " (Comtesse, 1890, pp. 7-9)

14. A titre d'exemple, $c f$. Hug (1902) et Krebs (1910).

15. Robert Comtesse, membre du parti radical suisse, sera au gouvernement cantonal neuchâtelois entre 1876-1899 et au gouvernement fédéral entre 1899 et 1912. (Cf. DHS). 
Ensuite, d'un point de vue socio-politique, ce mouvement semble préfigurer des changements sociétaux majeurs car, du prolétariat traditionnel en bleu de travail, actif dans les métiers de l'industrie et de l'artisanat, semble déjà se développer un "prolétariat en habit noir ", qui commence à accéder aux postes d'employés et aux professions libérales : un prolétariat qui, selon Léon Genoud, est " bien plus dangereux parce qu'il est mieux instruit " (1901, p. 408).

L'énonciation de ces deux ordres de préoccupation souligne l'exercice d'équilibriste entre progressisme et conservatisme auquel la filière doit se livrer : améliorer les compétences techniques, sans pour autant pousser les jeunes à revendiquer des changements sociaux majeurs. C'est d'ailleurs dans cette optique que les milieux bourgeois et patronaux vont réclamer, d'un côté, un enseignement le plus proche possible de l'exercice effectif d'une profession (d'où aussi la défense de l'apprentissage chez le patron) et, de l'autre, vont insister sur la nécessité de compléter cet enseignement par des cours de culture générale et d'éducation civique et morale promouvant le respect de l'ordre établi.

Les écoles professionnelles sont ainsi notamment invitées à introduire des enseignements dont les enjeux principaux sont, comme le relève Alfred Dufour, directeur de l'école d'arts et métiers de Genève, la transmission de "l'amour pour le travail " ou "le respect de l'ordre établi " et où " l'adolescent sera mis en garde contre les multiples tentations et les vices qui le guettent» (1920, p. 65). Toujours dans cette optique, l'enseignement de "culture générale ", en particulier d'histoire, de civisme ou d'économie, devient explicitement : "un excellent antidote à certaines théories que parfois nos jeunes gens sont appelés à entendre dans leurs ateliers " (Ernest Dubois, député libéral-démocratique vaudois, in $G d L, 26.02 .1925)$.

En bref, la formation professionnelle dans son double enseignement technique et de culture générale poursuit, au tournant du siècle, un but de stabilisation de la société qui se déploie aussi bien au niveau des conditions de vie des classes populaires qu'au niveau de leur contrôle social et professionnel ; le tout dans une volonté explicite d'augmenter l'acceptation, par les jeunes issus des classes populaires, de leur situation sociale, et d'œuvrer ainsi à une réduction des tensions sociales. Ainsi, comme on peut le lire dans le Journal de Genève, la formation professionnelle est appelée à jouer un rôle important :

"Une fois le travailleur armé pour le combat de la vie [c'est-à-dire bien formé], il se fera une idée plus juste de ses droits et de ses devoirs; il se vouera de cour et d'âme à la condition qu'il aura choisie. Il ne fuira pas les conseils de ceux qui non seulement veulent le relever à ses propres yeux par le sentiment de sa valeur et de la solidarité qui le lie à ses pairs; l'arracher aux suggestions de l'utopie ou des mauvais penchants, mais aussi lui inculquer le goût pour l'épargne et de l'assurance, en un mot le fortifier et l'encourager, aux jours difficiles, par la conscience de sa responsabilité et par la certitude du lendemain. " (JdG 27.11.1883) 
Mais encore plus : grâce à sa formation, un travailleur peut facilement devenir un médiateur, œuvrant directement pour la résolution des conflits dans le monde du travail :

"Le jeune qui en sort [des écoles professionnelles] trouve immédiatement sa place et en se trouvant, grâce à l'instruction reçue entre l'ouvrier inculte et le dirigeant, il sera le défenseur naturel des premiers, desquels il comprendra les justes aspirations, il en sera aussi le modérateur, car grâce à sa culture et sa pratique, il pourra facilement reconnaître le pour et le contre. " (Corriere del Ticino, 26.08.1902)

Pour conclure cette partie, revenons sur l'articulation entre finalités économiques et sociales telles qu'elles ont été attribuées à la formation professionnelle dès son origine. Cette articulation laisse en effet apparaître une convergence entre intérêts économiques liés à une amélioration des qualifications et intérêts sociaux de stabilisation de la société, une convergence qui permet de bien comprendre les raisons de l'engagement des milieux patronaux dans l'organisation d'un système de formation professionnelle publique : pour s'assurer, d'une part, de la main-d'œuvre qualifiée et disciplinée ${ }^{16}$ et, d'autre part, un cadre socioéconomique sans tension où développer les activités productives ou commerciales.

\section{Conclusion : de la stabilité à la mobilité}

Le parcours proposé ici nous a permis de comprendre le rôle que la formation professionnelle a été appelée à jouer, en lien avec la « question sociale » de l'époque. Dans la tentative de contribuer à l'atténuation des tensions socio-économiques qui font rage au tournant du siècle, cette filière s'est retrouvée à devoir gérer une mission complexe, tiraillée entre finalités sociales et économiques et entre éléments progressistes et éléments conservateurs : d'une part, assurer de la main-d'œuvre qualifiée à destination des entreprises suisses ; d'autre part, améliorer les conditions de vie des classes populaires, tout en participant à la stabilisation de la société et à la reproduction sociale afin d'éviter tout bouleversement socio-politique majeur.

Or, si en conclusion nous adoptons un point de vue contemporain sur la reconstruction historique proposée, ce qui nous frappe, ce n'est pas tant l'articulation entre finalités économiques et sociales, toujours présentes aujourd'hui encore, mais plutôt la tension interne aux finalités sociales de l'époque entre éléments progressistes et éléments conservateurs.

Une telle tension semble aujourd'hui avoir disparu. La volonté de stabilisation socio-professionnelle semble avoir laissé place, ces dernières décennies, à une volonté de promotion de la mobilité socio-professionnelle et de réduction des inégalités sociales.

16. Cf. la métaphore de l' "armée d'ouvriers qualifiés " qui apparait souvent dans les textes des milieux bourgeois de l'époque, à titre d'exemple : Comtesse (1890) et Krebs (1910). 
Au cours de la période analysée dans les pages précédentes, rares sont les signes avantcoureurs de ce changement, si ce n'est l'évocation d'un certain "esprit d'émancipation", fortement critiqué, qui prend progressivement forme parmi les jeunes ${ }^{17}$.

Il faudra attendre la fin de la seconde Guerre mondiale pour que ces aspirations soient reconnues et légitimées dans des discours officiels autour de ce type de formation. En témoigne le Message du Conseil fédéral concernant la deuxième loi sur la formation professionnelle de 1962, où apparaissent pour la première fois des expressions comme " avancement professionnel» (p. 873) (beruficher Aufstieg) ou "avancement social» (p. 875) (soziale Aufstieg).

Mais c'est surtout à partir des années 1990 que la nouvelle orientation va se traduire par des mesures concrètes destinées à promouvoir la mobilité sociale, l'intégration professionnelle et la réduction des différences sociales. Ces nouvelles mesures visent notamment à relancer l'attractivité de la filière professionnelle en l'intégrant dans le système de l'éducation générale $^{18}$, avec pour corollaire la mise en place d'une Maturité professionnelle ${ }^{19}$ et d'une série de passerelles permettant une grande perméabilité entre les différents niveaux du système et entre filière générale (lycées et universités) et filière professionnelle ${ }^{20}$. Parallèlement, ces aménagements ont aussi visé une meilleure et plus grande intégration des jeunes dans la filière professionnelle, avec notamment des mesures favorisant l'intégration des jeunes dits à risque ${ }^{21}$.

17. En particulier dans les ouvrages sur l'orientation professionnelle des jeunes, à titre d'exemple : Hug (1902) et Krebs (1910).

18. La volonté d'intégrer la formation professionnelle dans le système éducatif suisse est un des points saillants de la loi fédérale adoptée en 2002, cf. notamment Message du Conseil fédéral de 2000, ch. 1.6.

19. La Maturité professionnelle (MP) est un cursus formatif complémentaire au diplôme de formation professionnelle initiale (CFC) donnant lieu à un diplôme de " Maturité professionnelle fédérale » qui ouvre l'accès aux formations supérieures au niveau tertiaire, dans des hautes écoles spécialisées. Pour une présentation de la MP $c f$. http://www.sbfi.admin.ch/themen/01366/01379/01570/index.html?lang=fr. (12.02.2016). Les raisons de politique formative à l'origine de la MP sont présentées dans Gonon (2013).

20. Sur la mise en place de ces passerelles, voir les informations fournies sur les pages internet du Secrétariat d'Etat à la formation, la recherche et l'innovation (SEFRI) : http://www.sbfi.admin.ch/themen/01366/01379/01629/index.html?lang=fr (12.02.2016).

21. La notion de " jeunes à risque " renvoie à un groupe de jeunes qui éprouve des difficultés d'insertion formative ou professionnelle après l'école obligatoire et notamment qui "passe entre les mailles du système [formatif] et n'obtient pas de formation qualifiée au degré secondaire II [... laissant] entrevoir des problèmes sociaux et économiques sur le long terme pour la société (risque de chômage, mise à l'invalidité et dépendance de l'assistance sociale)" (Häfeli \& Schellenberg 2009, p. 14). Différentes mesures ont été prises pour faciliter l'accès à une formation professionnelle pour ce groupe de jeunes. Notamment : l'introduction d'un diplôme de deux ans (Attestation fédérale de formation professionnelle - AFP) moins exigeant que celui traditionnel en trois ou quatre ans, ( $c f$. Message du Conseil Fédéral de 2000, ch. 1.7.2) ; et la mise en place de mesures d'accompagnement pour les jeunes ayant des difficultés d'insertion, $c f$. les pages internet du SEFRI consacrées au Case Management Formation professionnelle (http://www.sbfi.admin.ch/berufsbildung/01496/ index.html?lang=fr (12.02.2016). 
Cependant, cette nouvelle orientation sociale renforce aussi la tension entre finalités économiques et finalités sociales, laissant notamment apparaître une série de contradictions, en relation directe avec les intérêts des différents acteurs en jeu et avec la prédominance de l'apprentissage en alternance qui caractérise le système suisse.

Parmi ces contradictions, on mentionnera, d'une part, la volonté politique de promouvoir la formation et la spécialisation individuelle et, d'autre part, le besoin des entreprises de disposer d'une main-d'œuvre peu qualifiée et à bon marché2 ${ }^{2}$. On notera encore la volonté d'intégrer le plus grand nombre de jeunes dans l'apprentissage, et en particulier les jeunes issus de groupes dits à risque ou rencontrant des difficultés scolaires ; les exigences toujours plus élevées des entreprises vis-à-vis des compétences des jeunes dans l'optique de les embaucher comme apprentis ${ }^{23}$; enfin, la volonté politique d'offrir une formation professionnelle qui privilégie les compétences transversales favorisant la mobilité professionnelle, de même que les exigences du monde du travail qui s'orientent vers une formation de plus en plus spécifique à telle profession sinon à telle activité au sein de telle entreprise ${ }^{24}$.

Or, ces contradictions émergent d'un système dont les fondations ont été établies il y a cent ans, et dont les finalités sociales ont été transformées ces dernières décennies pour se retrouver complètement renversées par rapport aux finalités d'origine.

Ainsi, l'engagement des entreprises et des associations professionnelles dans un système public de formation professionnelle promouvant la stabilité et la reproduction des différences sociales était facilement compréhensible, en raison notamment de la convergence entre intérêts économiques et intérêts sociaux relevée auparavant. Aujourd'hui, il est en revanche plus difficile de comprendre l'engagement de ces milieux dans un système qui promeut la mobilité et la réduction des inégalités sociales. Le risque serait celui d'une exacerbation de la tension entre finalités économiques et finalités sociales qui pourrait conduire le monde du travail à délaisser ces dernières au profit des premières.

Mais la perspective historique développée ici ouvre aussi une autre piste de réflexion : compte tenu du soutien des milieux économiques suisses dont bénéficie, aujourd'hui encore, la formation professionnelle, dans ses deux finalités, on pourrait se demander si une telle convergence n'est tout de même pas encore présente. De même, la volonté de

22. Ce besoin se reflète dans les cas, régulièrement dénoncés par les associations syndicales, où les entreprises profitent de l'apprentissage pour recruter de la main-d'oeuvre à bas coût, $c f$. Polito, 2014.

23. Selon le Baromètre des places d'apprentissage de 2015, les qualifications inadéquates des candidats sont à l'origine de plus de $60 \%$ des places d'apprentissages non repourvues (Linkinstitut, 2015).

24. L'équilibre entre une formation professionnelle générale ou bien une formation spécifique est toujours au centre de négociations entre des acteurs du système de la formation professionnelle suisse. Avec plus de 230 professions reconnues et les trois quarts de la formation réalisés en entreprise, la formation professionnelle suisse est ainsi orientée vers les besoins spécifiques des entreprises ( $c f$. Maurer \& Pieneck, 2013 p. 82). Néanmoins, il résulte aussi que les compétences générales acquises suffisent pour assurer une certaine mobilité aux diplômés : plus de 50 \% des personnes possédant un CFC exercent une profession différente de celle apprise, (cf. Cattaneo, 2010) sans que cela se traduise par une baisse salariale (Eymann et Schweri, 2015). 
mobilité et de réduction des inégalités sociales d'aujourd'hui comme celle de reproduction sociale d'il y a cent ans, ne répondent-elles pas, au fond, sous des conditions socio-économiques différentes, aux mêmes finalités de stabilisation du contexte socio-économique et de mise à disposition d'une main-d'œuvre qualifiée et disciplinée ?

\section{Bibliographie}

\section{Sources citées}

Arbeiterstimme (Ast), 1881-1908.

Ascapa (Association Suisse de Conseils d'Apprentissage et de Protection des Apprentis). (1920), Orientation professionnelle. Conférences du 24-25.09.1920 à l'Université de Genève, Genève, Meyer.

Comtesse R. (1890), La question des apprentissages, Neuchâtel, Borel.

Conseil Fédéral (CF) (1883), « Message du Conseil fédéral à l'Assemblée fédérale sur l'enquête industrielle (du 3 décembre 1883) ", Feuille Fédérale (08.12), 4(62), pp. 613-724.

Conseil Fédéral (CF) (1962), " Message du Conseil fédéral à l'Assemblée fédérale concernant un projet de loi sur la formation professionnelle (du 28 septembre 1962) ", Feuille Fédérale (01.11), 2(44), pp. 865-966.

Corriere del Ticino (CdT), 1880-1930.

Dufour A. (1920), "L'apprentissage à l'Ecole », In Accapa (Ed.), Orientation professionnelle, pp. 53-66), Genève, Eugène Meyer.

Droz N. (1884), Instruction civique. Manuel à l'usage des écoles primaires supérieures, des écoles secondaires, des écoles complémentaires et des jeunes citoyens, Lausanne, Daniel Lebet.

Gazette de Lausanne (GdL), 1880-1930.

Genoud L. (1901), L'enseignement professionnel. L'enseignement à l'exposition universelle de Paris 1900, Fribourg, Imprimerie de l'œuvre de Saint-Paul.

Hug G. (1902), Le choix d’une profession, Berne, Librairie-imprimerie Büchler \& Co.

Journal de Genève (JdG), 1880-1930.

Krebs W. (1910), Méthode pour le choix d’une profession, Lausanne, Fédération suisse des Patronats d'Apprentissage, Imprimerie Bridel.

Littérature secondaire

Bauder T. (2008), „Der Entwicklungsprozess des ersten eidgenössischen Berufsbildungsgesetzes. Unterschiedliche Interessen, gemeinsames Ziel“, in Bauder T. 
\& Osterwalder F. (Eds.), 75 Jahre eidgenössisches Berufsbildungsgesetz, pp. 11-57, Bern, Hep.

Bonoli L. (2012), " La Naissance de la formation professionnelle en Suisse : entre compétences techniques et éducation morale ", Education permanente (192), pp. 209-221.

Cattaneo A. (Ed.) (2010), Scuola a tutto campo. Gli indicatori del sistema educativo ticinese, Locarno, SUPSI-DFA.

Cedefop (Ed.). (2004), Towards a history of vocational education and training (VET) in Europe in a comparative perspective, Luxemburg, Office for Official Publications of the European Communities.

Conseil Fédéral (CF) (2000), "Message relatif à une nouvelle loi sur la formation professionnelle (00.072)", Feuille Fédérale (47), pp. 5256-5350.

Dictionnaire Historique Suisse (DHS), consultation online : http://www.hls-dhs-dss.ch/ index.php.

Foucault M. (1966), Les mots et les choses, Paris, Gallimard.

Foucault M. (1969), L'archéologie du savoir, Paris, Gallimard.

Fuessel M. \& Neu T. (2014), „Diskursforschung in der Geschichtswissenschaft“, in Angermueller J. \& et alii (Eds.), Diskursforschung. Ein interdiziplinäres Handbuch, Vol. 1, , Bielefeld, transcript Verlag.

Gonon P. (1998), „Berufliche Bildung zwischen Zunft, Handelsfreiheit und Demokratie“, Schweizerische Zeitschrift für Erziehungswissenschaft, 3, pp. 419-429.

Gonon P. (2013), „Berufsmaturität als Reform - Hybris oder Erfolgsstory?“, in Maurer M. \& Gonon P. (Eds.), Herausforderungen für die Berufsbildung in der Schweiz, pp. 119146, Berne, HEP.

Gruner E. (1968), Die Arbeiter in der Schweiz im 19. Jahrhundert. Soziale Lage, Organisation, Verhältnis zu Arbeitgeber und Staat, Bern, Frank.

Haefeli K. \& Schellenberg C. (2009), Facteurs de réussite dans la formation professionnelle des jeunes à risque, Berne, EDK-CDIP.

Hirter H. (1983), Die Arbeitskämpfe in der Schweiz von 1880 bis 1914 . Eine quantitative Streikanalyse, Bern, Doktorarbeit.

Jaeger S. (2012), Kritische Diskuranalyse. Eine Einführung. Münster, Unrast-Verlag.

Keller R. (2011), Diskursforschung. Eine Einführung für SozialwissenschaftlerInnen, Wiesbaden, VS Verlag. 
Lamamra N. (2013), "Entre produire et former : Tension entre éducation générale et préparation à l'entrée sur le marché du travail dans la formation professionnelle suisse ", Questions d'orientation, 4. Actes du congrès international d'orientation de Montpellier, pp. 74-82.

Linkinstitut (2015), Baromètre des places d'apprentissage. Sur mandat du SEFRI, Bern, OFFT.

Maingueneau D. (1991), L'analyse du Discours. Introduction aux lectures de l'archive, Paris, Hachette.

Maurer M. \& Pieneck S. (2013), „Die Reform von Berufsbildern als ständige Auseinandersetzung über Form und Inhalt", In Maurer M. \& Gonon P. (Eds.), Herausforderungen für die Berufsbildung in der Schweiz, pp. 81-100, Bern, hep.

Moreau G. (2003), Le monde apprenti, Paris, La Dispute.

Pelpel P. \& Troger V. (2001), Histoire de l'enseignement technique, Paris, L'Harmattan.

Polito V. (2014), Le Conseil fédéral ignore les problèmes touchant les apprenti(e)s, communiqué de presse paru sur http://www.uss.ch/ (20.01.2015).

Sefri (Secrétariat d'Etat à la formation, la recherche et l'innovation) (2013), La formation professionnelle en Suisse. Faits et données chiffrées, Berne, SEFRI.

Surdez M. (2005), Diplôme et nation. La constitution d'un espace suisse des professions avocates et artisanales, Berne, Peter Lang.

Tabin J.-P. (1989), Formation professionnelle en Suisse. Histoire et Actualité, Lausanne, Réalités sociales.

Usam Union suisse des arts et métiers / SGV (Schweizerischer Gewerbeverein) (1881), Das Gewerbliche Lehrlingswesen. Zwei Preisschriften, auf Veranlassung des schweizerischen Gewerbevereins, Winterthur, Westfehling.

Widmer Th. (1992), Die Schweiz in der Wachstumskrise der 1880er Jahre, Zürich, Chronos. 